\title{
Mapping Speech Spectra from Throat Microphone to Close-Speaking Microphone: A Neural Network Approach
}

\author{
A. Shahina ${ }^{1}$ and B. Yegnanarayana ${ }^{2}$ \\ ${ }^{1}$ Department of Computer Science and Engineering, Indian Institute of Technology Madras, Chennai 600036, India \\ ${ }^{2}$ International Institute of Information Technology, Gachibowli, Hyderabad 500032, India
}

Received 4 October 2006; Accepted 25 March 2007

Recommended by Jiri Jan

Speech recorded from a throat microphone is robust to the surrounding noise, but sounds unnatural unlike the speech recorded from a close-speaking microphone. This paper addresses the issue of improving the perceptual quality of the throat microphone speech by mapping the speech spectra from the throat microphone to the close-speaking microphone. A neural network model is used to capture the speaker-dependent functional relationship between the feature vectors (cepstral coefficients) of the two speech signals. A method is proposed to ensure the stability of the all-pole synthesis filter. Objective evaluations indicate the effectiveness of the proposed mapping scheme. The advantage of this method is that the model gives a smooth estimate of the spectra of the close-speaking microphone speech. No distortions are perceived in the reconstructed speech. This mapping technique is also used for bandwidth extension of telephone speech.

Copyright (C) 2007 A. Shahina and B. Yegnanarayana. This is an open access article distributed under the Creative Commons Attribution License, which permits unrestricted use, distribution, and reproduction in any medium, provided the original work is properly cited.

\section{INTRODUCTION}

Speech signal collected by a vibration pickup (called throat microphone) placed at the throat (near the glottis) is clean, but does not sound natural like a normal (close-speaking) microphone speech. Mapping the speech spectra from the throat microphone to the normal microphone aims at improving the perceptual quality of the slightly muffled and "metallic" speech from the throat microphone. This would reduce the discomfort arising due to prolonged listening to speech from a throat microphone in adverse situations as in cockpits of aircrafts, in the presence of intense noise of running engines at machine shops and engine rooms among others, where it is currently used.

Mapping the speech spectra involves the following stages: the first stage consisting of training involves recording speech simultaneously using the throat microphone and normal microphone from a speaker. Simultaneous recording is essential for understanding the differences between components of speech in both signals and for training appropriate models to capture the mapping between the spectra of the two signals. Suitable speech features are extracted from the speech signals. During training, the feature vectors extracted from the throat microphone (TM) speech are mapped onto the corresponding feature vectors extracted from the normal microphone (NM) speech. In the second stage consisting of testing, feature vectors corresponding to the NM speech are estimated for each frame of the TM speech. The estimated features are used to reconstruct the speech.

Two major issues are addressed in the approach proposed in this paper: (a) a suitable mapping technique to capture the functional relationship between the feature vectors of the two types of speech signals, and (b) an approach to ensure that the estimated feature vectors generated by the model result in a stable all-pole filter for synthesis of speech.

The TM speech is typically a low bandwidth signal, whereas the NM speech is of wide bandwidth. Since both speech signals are recorded simultaneously from the same speaker, it is assumed that the TM speech and the NM speech are closely related. The problem of mapping then can be viewed as mapping of the low-bandwidth (throat) signal to the corresponding high-bandwidth (normal) signal. There exist a variety of approaches in the literature dealing with the issue of bandwidth extension of telephony speech [1-3], which has a low bandwidth (300 to $3400 \mathrm{~Hz}$ ). The motivation for telephony speech has been to increase the bandwidth to improve its pleasantness at the receiving end. The procedure involves constructing the wideband residual signal (referred 
to as high-frequency regeneration) and determining a set of wideband linear prediction (LP) coefficients. Once these two components are generated, the wideband residual is fed to the wideband synthesis filter derived from the wideband LP coefficients to produce a wideband speech signal.

The commonly adopted high-frequency regeneration methods are $[1,4]$ (a) rectification of the upsampled narrowband residual to generate high-frequency spectral content, followed by filtering through an LP analysis filter to generate spectrally flat residual, (b) spectral folding, which involves the expansion of the narrowband residual through the insertion of zeros between adjacent samples, and (c) spectral shifting, where the upsampled narrowband residual is multiplied by a cosine function resulting in a shift in the original spectrum.

There are several approaches for the reconstruction of the wideband spectrum. Codebook mapping is one approach which relies on a one-to-one mapping between the codebooks of narrowband and wideband spectral envelopes $[1,5,6]$. During the testing phase, for each frame of the narrowband speech, the best fitting entry of the wideband codebook is selected as the desired estimate of the wideband spectral envelope. Statistical approaches such as Gaussian mixture models (GMM) and hidden Markov models (HMM) used for the wideband spectral estimation were reported to provide smooth classification indices, thereby avoiding unnatural discontinuities prevalent in VQ-based approaches $[7,8]$. Neural network approaches that use a simple nonlinear mapping from narrow to wideband speech signal have been exploited to estimate the missing frequency components $[9,10]$. The stability of the all-pole filter derived from the network output is important for synthesis. To ensure this in the all-pole filter, poles existing outside the unit circle if any were reflected within the unit circle.

Alternate speech sensors have been used to estimate feature vectors of clean close-talking microphone speech. In [11], throat microphone and normal microphone were used in combination to increase the robustness of speech recognizers. Noisy mel-cepstral features from the normal and throat microphones, juxtaposed as an extended feature vector, were mapped to mel-cepstral feature vectors of clean normal microphone speech. In [12], a bone-conductive sensor, integrated with a close-talking microphone, was used to enhance the wideband noisy speech for use with an existing speech recognition system. A mapping from the bone sensor signal to the clean speech signal was learnt, and then the bone signal and the noisy signal were combined to obtain the final estimate of the clean speech. In the above two studies, the alternate speech sensor has been used in combination with a normal microphone to obtain clean speech. In our study, we estimate the features of the normal microphone speech from the features of the throat microphone speech alone. This is useful in situations where the throat microphone alone is used by the speakers.

In this paper, a multilayered feedforward neural network is used to capture the functional relationship between the features of the TM speech and NM speech of a speaker. We propose an approach that uses autocorrelation method to derive the coefficients of a stable all-pole filter [13]. The advantage of the proposed method is that no discontinuity is perceived between successive frames of the reconstructed speech. This is because the network provides a smooth estimate of the wideband normal spectra.

The paper is organized as follows: Section 2 gives a description of the spectral characteristics of the TM speech in comparison with those of the NM speech. The proposed method for spectral mapping from the TM speech to the NM speech is detailed in Section 3. The features and the mapping network used for capturing the functional relationship between the TM speech and the NM speech are explained. This section also discusses the behavior of the network in capturing the mapping for different types of sound units, and illustrates the efficiency of mapping during testing. An objective measure is used to assess the quality of the regenerated speech. In this section, it is also shown that the mapping technique can be effectively used to extend the bandwidth of narrowband telephone speech. Section 4 summarizes this work and lists some possible extensions.

\section{SPECTRAL CHARACTERISTICS OF TM SPEECH AND NM SPEECH}

The perceptual differences between the TM speech and the NM speech depend on their acoustic characteristics. This section describes a comparative acoustic analysis of various sound units in the two speech signals based on the analysis of their acoustic waveforms, spectrograms, linear prediction spectra derived from the closed-glottis regions after the instants of significant excitation [14], and pitch synchronous formant trajectories of syllables. The pitch synchronous analysis provides an accurate estimate of the frequency response of the vocal tract system.

Five broad categories of sound units, namely, vowels, stops, nasals, fricatives, and semivowels of the Indian language (Hindi) are studied. In the case of vowels, the lower formants are spectrally well defined in the TM speech, as in the NM speech. However, most of the higher frequencies (above $3000 \mathrm{~Hz}$ ) are missing in TM speech. This can be observed in the LP spectra derived from the closed-glottis regions of the vowels as shown in Figure 1. The formant locations of the back vowels in the two signals vary. For example, in the case of back vowel $/ u$ / in the NM speech, the second formant is lowered due to the effect of lip rounding. The first and second formants are close, indicating the backness of the vowels. But in the TM speech, the second formant is high like in the front vowels. Figure 1 shows that the spectra of vowel $/ u$ / resemble that of vowel $/ i$ / in the TM speech. This increases the confusability between the two vowels. Consequently, recognition of these two vowels is poorer in the case of TM speech as compared to the NM speech [15].

In voiced stop consonants, the closure is characterised by (low frequency) energy in the 0 to $500 \mathrm{~Hz}$ range for NM speech. The vocal fold vibration accompanying the closure is perceived as low frequency since the normal microphone picks up the vibration during the closure phase as it propagates through the walls of the throat. This activity is referred 


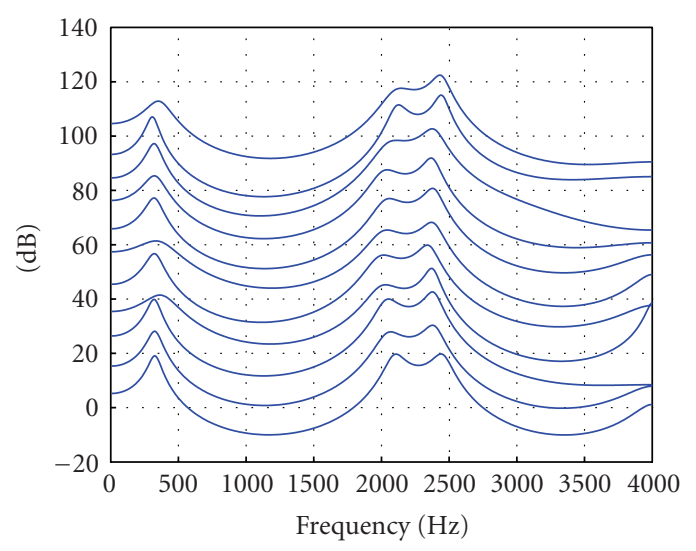

(a)

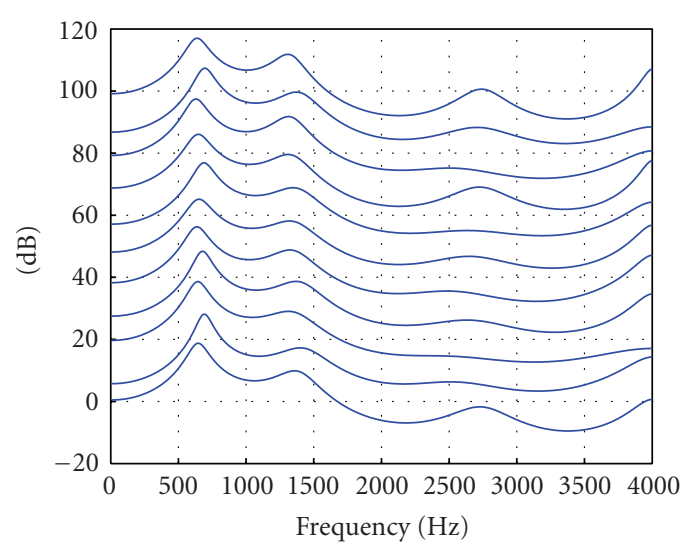

(b)

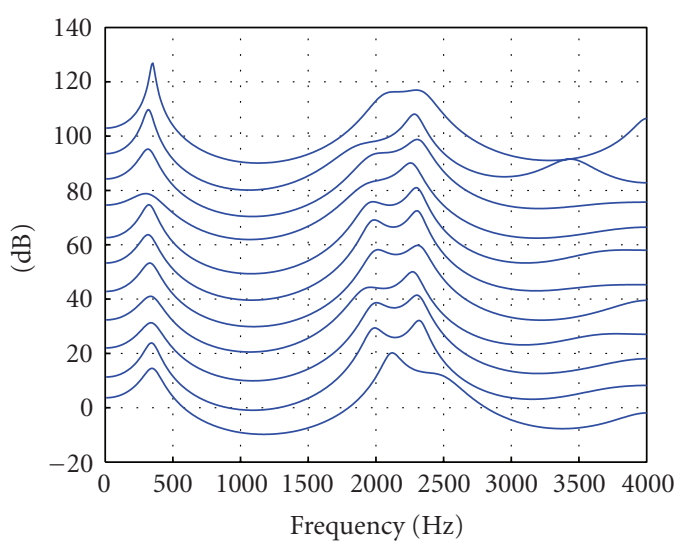

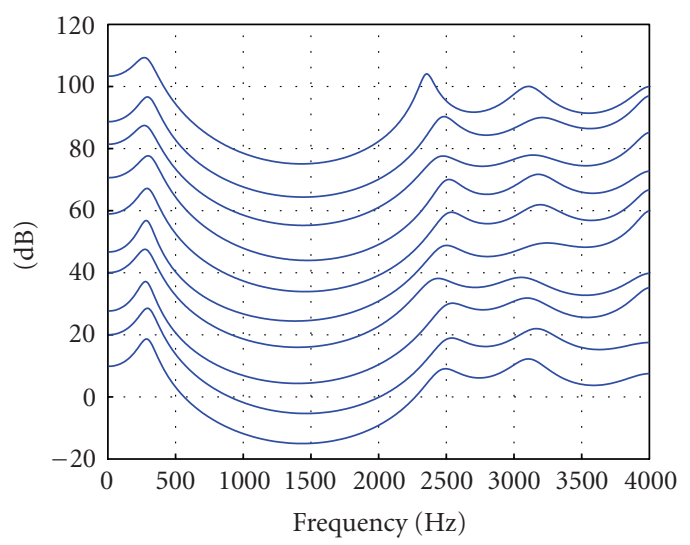
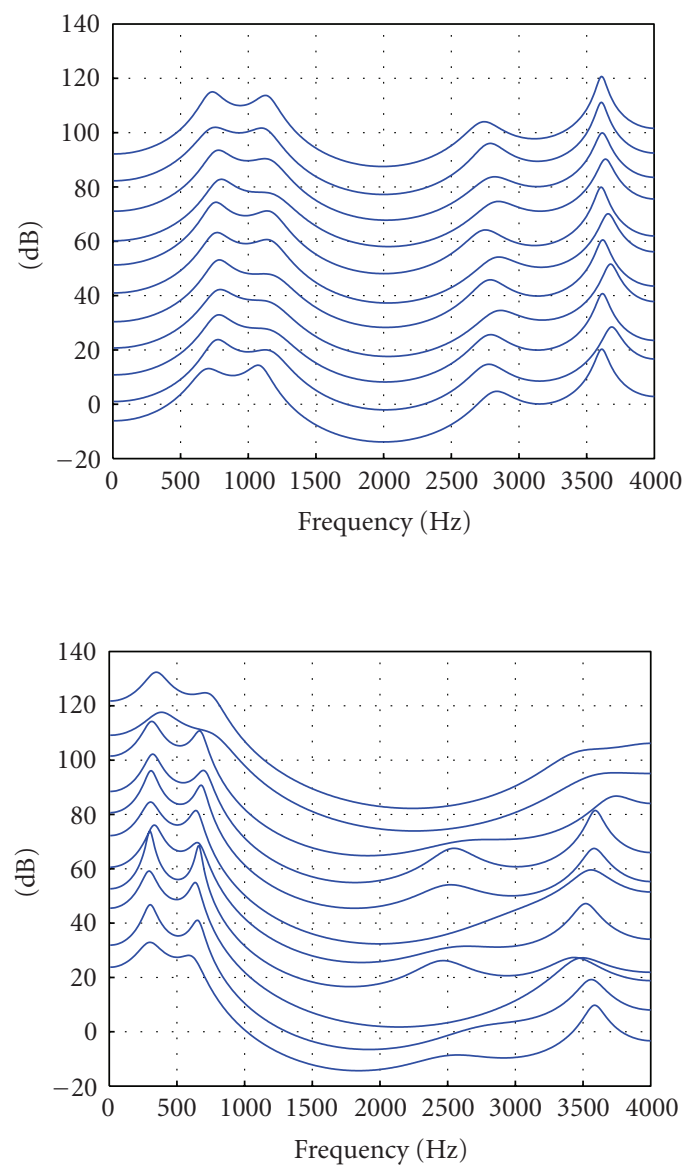

(c)

FigURE 1: LP spectra of 11 successive closed-glottis regions of (a) front vowel /i/, (b) mid vowel /a/, and (c) back vowel / u/ from simultaneously recorded TM speech and NM speech.

to as the "voice bar" [16]. However, in the TM speech, the closure region of each of the voiced stops is characterised by distinct well-defined formant-like structures. This is due to the placement of the throat microphone close to the vocal folds. It picks up the resonances of the oral cavity (behind the region of closure) associated with the vibrations of the vocal folds during the closure of the voiced stop consonants.
These distinct formant-like structures in the TM speech serve as acoustic cues that can be used to resolve the highly confusable voiced stops into classes based on the place of articulation [15].

Nasal consonants in the NM speech are characterised by distinct low amplitude, damped periodic waveforms. This is because during the production of nasals the oral cavity is 
TABLE 1: Characteristics of sound units in TM speech and NM speech.

\begin{tabular}{lll}
\hline Characteristics of sound units & Normal speech & Throat speech \\
\hline Energy distribution & Up to Nyquist frequency & $<3000 \mathrm{~Hz}$ \\
Formant bandwidth & Narrow & Large \\
Formant location of back vowels & Low second formant & High second formant like front vowels \\
Closure phase of voiced stop consonants & Low frequency “voice-bar" & Formant-like structures \\
$\begin{array}{l}\text { Aspiration phase of stop consonants } \\
\text { Signal damping in nasal consonants }\end{array}$ & Large amplitude noise & Low-amplitude noise \\
$\begin{array}{l}\text { Intensity of formants in semivowels and } \\
\text { nasal consonants }\end{array}$ & Highly damped & Less damped like vowels \\
Formant locations of nasal consonants & Less compared to vowels & Similar to vowels \\
\hline
\end{tabular}

completely closed at some location, and the sound is radiated through the nostrils. The damping in the nostrils affects the relative amplitude of the nasals. In contrast, in the TM speech, the effect of damping is minimal. So, the waveforms of nasals appear more like vowels. Distinct formant locations characteristic of the nasals are seen in both the TM and NM speeches. While the lower-formant locations are similar in both the TM and NM speeches, the higher-formant locations differ. This could be due to the resonances of the oral tract appearing in the TM speech.

Fricatives $\left(/ s /, / \int /, / h /\right)$ in the NM speech signal are characterised by the presence of energy distributed over a wide range of frequencies extending even beyond $8000 \mathrm{~Hz}$. In the TM speech, fricatives are characterised by the distribution of the noise energy restricted to a band of frequencies between 2000 and $3500 \mathrm{~Hz}$. This is because the turbulence in the airflow caused by the constriction in the oral tract is not as effectively captured by the throat microphone as compared to the normal microphone.

For semivowels, in the NM speech the formants have a lower intensity than the vowels, with an abrupt change in intensity observed at the transition from semivowel to vowel (or vice versa). In the TM speech, the intensity of the formants of the semivowels is similar to that of the vowels, and hence there is no abrupt change at the transition region from semivowel to vowel or vice versa.

Some of the differences in the acoustic characteristics between the TM speech and NM speech for various sound units are summarized in Table 1.

\section{MAPPING SPECTRAL FEATURES OF TM SPEECH TO NM SPEECH}

The study of the acoustic characteristics of TM and NM speeches brings out the differences in the spectra of the two speech signals for various sound units. These differences could be one of the contributing factors for the unnaturalness of the TM speech. In order to improve the perceptual quality of the TM speech, we need to compensate for these differences in the spectra.

The focus in this paper is (a) to achieve an effective mapping between the spectral features of the TM and NM speeches, (b) to ensure that the all-pole synthesis filter de- rived from the learnt mapping is stable, and (c) to ensure that the synthesized speech does not suffer from discontinuities due to spectral "jumps" between adjacent frames. The filter for synthesis is obtained by (1) using the cepstral coefficients from both the TM and NM speech signals for initially training a mapping network, and (2) deriving an all-pole filter from the estimated cepstral coefficients that are obtained from the trained mapping network. The method of deriving the synthesis filter is described below.

\subsection{Features for mapping}

Cepstral coefficients are used to represent the feature vector of each frame of data. The cepstral coefficients are derived from the LP coefficients. The cepstral coefficients are obtained from the LP spectrum as follows [17].

The LP spectrum for a frame of speech is given by

$$
|H(k)|^{2}=\left|\frac{1}{1+\sum_{n=1}^{p} a_{n} e^{-j(2 \pi / M) n k}}\right|^{2}, \quad k=0,1, \ldots, M-1,
$$

where $a_{n} s$ are the LP coefficients, $M$ is the number of spectral values, and $p$ is the LP order. The inverse discrete Fourier transform (DFT) of the log LP spectrum gives the cepstral coefficients $c_{n}$. Let

$$
S(k)=\log |H(k)|^{2} \text {. }
$$

Then

$$
c_{n}=\frac{1}{M} \sum_{k=0}^{M-1} S(k) e^{j(2 \pi / M) k n}, \quad n=0,1, \ldots, M-1 .
$$

Only the first $q$ cepstral coefficients are chosen to represent the LP spectrum. Normally, $q$ is chosen much larger than $p$ in order to represent the LP spectrum adequately.

Linearly weighted cepstral coefficients $n c_{n}, n=1,2, \ldots$, $q$, are chosen as a feature vector representing the frame of speech. The weighted linear prediction cepstral coefficients (wLPCCs) are derived for each frame of the throat speech and for the corresponding frame of the NM speech. These pairs of wLPCCs vectors are used as input-output pairs to 
train a neural network model to capture the implicit mapping.

In the testing stage, the output of the trained network for each frame of the TM speech of a test utterance gives an estimate of the wLPCCs of the corresponding frame of NM speech. The wLPCCs are deweighted. From these estimated LPCCs $\hat{c}_{n}, n=1,2, \ldots, q$, the estimate of the log LP spectrum is obtained by performing DFT. Let $\hat{S}(k), k=0,1,2, \ldots, M-$ 1 , be the estimated log spectrum. The estimated spectrum $\hat{P}(k)$ is obtained as

$$
\widehat{P}(k)=e^{\hat{S}(k)}, \quad k=0,1,2, \ldots, M-1 .
$$

From the spectrum $\hat{P}(k)$, the autocorrelation function $\hat{R}(n)$ is obtained using inverse DFT of $\widehat{P}(k)$.

The first $p+1$ values of $\widehat{R}(n)$ are used in the LevinsonDurbin algorithm to derive the LP coefficients. These LP coefficients for each frame are used to resynthesize the speech by exciting the time-varying filter with the LP residual of the TM speech. The all-pole synthesis filter derived from these LP coefficients is stable because they are derived from the autocorrelation function.

\subsection{Neural network model for mapping spectral features}

Given a set of input-output pattern pairs $\left(\mathbf{a}_{l}, \mathbf{b}_{l}\right), l=$ $1,2, \ldots, L$, the objective of pattern mapping is to capture the implied mapping between the input and output vectors. Once the system behavior is captured by the neural network, the network would produce a possible output pattern for a new input pattern not used in the training set. The possible output pattern would be an interpolated version of the output patterns corresponding to the input training patterns which are closest to the given test input pattern $[18,19]$. The network is said to generalize well when the input-output mapping computed by the network is (nearly) correct for the test data that is different from the examples used to train the network [20]. A multilayered feedforward neural network (MLFFNN) with at least two intermediate layers in addition to the input and output layers can perform a pattern mapping task [18]. The additional layers are called the hidden layers. The neurons in these layers, called the hidden neurons, enable the network to learn complex tasks by extracting progressively more meaningful features from the input pattern vectors. The input and output neurons for this task are linear units, while the hidden neurons are nonlinear units. The activation function of the hidden neurons is continuously differentiable to enable the backpropagation of error.

The mapping between the training pattern pairs involves iteratively determining a set of weights $\left\{w_{i j}\right\}$ such that the actual output $\mathbf{b}_{l}^{\prime}$ is equal (or nearly equal) to the desired output $\mathbf{b}_{l}$ for all the given $L$ pattern pairs. The weights are determined by using the criterion that the total mean squared error between the desired output and the actual output is to be minimized. The total error $E$ over all the $L$ input-output

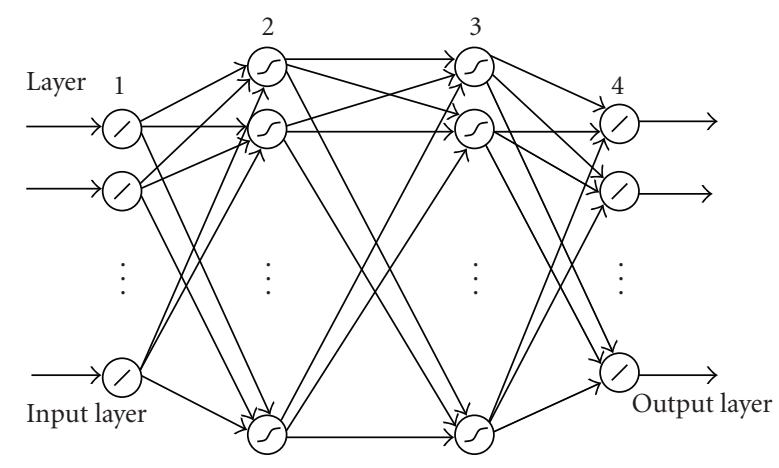

FIGURE 2: A 4-layer mapping neural network of size 12L $24 \mathrm{~N} 24 \mathrm{~N}$ $12 L$, where $L$ refers to a linear unit and $N$ to a nonlinear unit, the numbers represent the number of nodes in a layer.

pattern pairs is given by

$$
E=\frac{1}{L} \sum_{l=1}^{L}\left\|\mathbf{b}_{l}-\mathbf{b}_{l}^{\prime}\right\|^{2} .
$$

To arrive at an optimum set of weights to capture the mapping implicit in the set of input-output pattern pairs, and to accelerate the rate of convergence, the conjugate gradient method is used. In the conjugate gradient method, the increment in weight at the $(m+1)$ th iteration is given by

$$
\Delta \mathbf{w}=\eta(m) \mathbf{d}(m),
$$

where $\eta$ is the learning rate parameter. The direction of increment $\mathbf{d}(m)$ in the weight is a linear combination of the current gradient vector and the previous direction of the increment in the weight [18]. That is,

$$
\mathbf{d}(m)=-\mathbf{g}(m)+\alpha(m-1) \mathbf{d}(m-1),
$$

where $\mathbf{g}(m)=\partial E / \partial \mathbf{w}$. The value of $\alpha(m)$ is obtained in terms of the gradient using the Fletcher-Reeves formula given by

$$
\alpha(m)=\frac{\mathbf{g}^{T}(m+1) \mathbf{g}(m+1)}{\mathbf{g}^{T}(m) \mathbf{g}(m)} .
$$

The objective is to determine the value of $\eta$ for which the error $E[\mathbf{w}(m)+\mathbf{d}(m)]$ is minimized for the given values of $\mathbf{w}(m)$ and $\mathbf{d}(m)$.

\subsection{Experimental results}

The training and testing data are obtained from the same speaker because the mapping is speaker-dependent. The simultaneously recorded speech signals from a throat microphone and a normal microphone are sampled at a rate of $8 \mathrm{kHz}$. For training, 5 minutes of speech data (read from a text, and containing speech as well as nonspeech regions) are used. LP analysis is performed on Hamming windowed speech frames, each of 20 millisecond duration. The overlap between adjacent frames is 5 milliseconds. The wLPCCs are derived from the TM speech and the NM speech. After experimenting with several LP orders, an LP order of $p=8$ and 

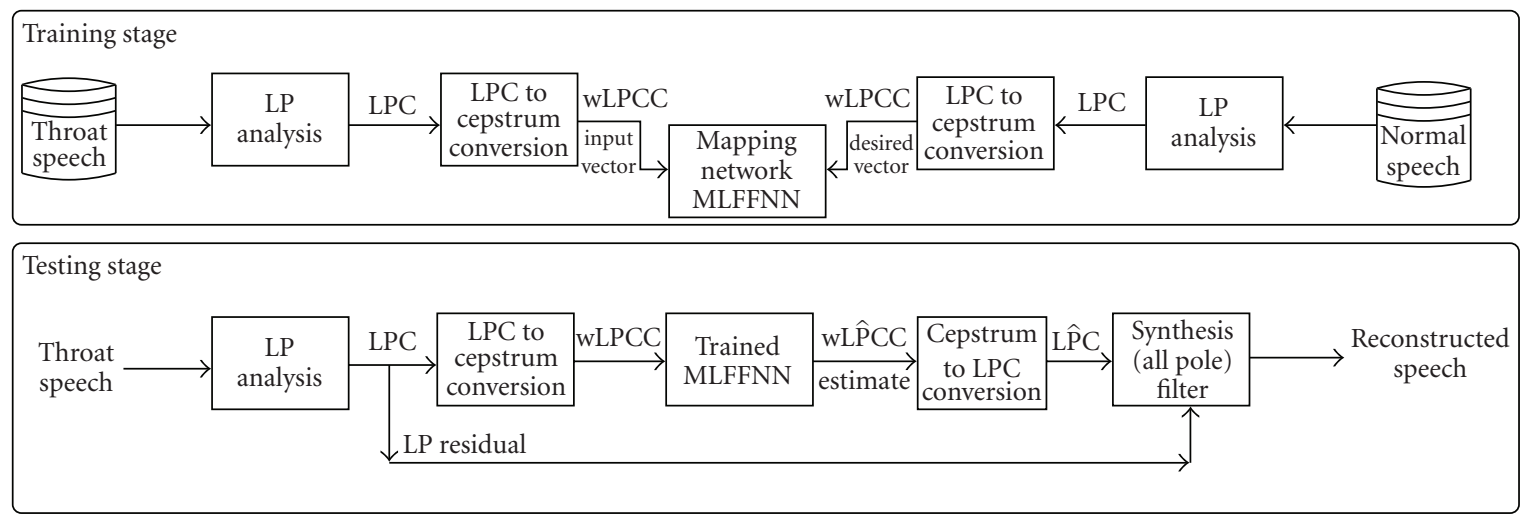

FIGURE 3: Block diagram of the proposed approach for modeling the relationship between the TM speech and the NM speech of a speaker.

the number of wLPCCs $q=12$ are chosen, although these choices are not critical. Each training pattern is preprocessed so that its mean value, averaged over the entire training set, is close to zero. Each pattern (vector) is normalized so that the component values fall within the range $[-1,1]$. This accelerates the training process of the network [20]. These preprocessed wLPCCs derived from the TM speech and the NM speech form the input-output training pairs, respectively, for the mapping network. The training pattern pairs are presented to the network in the batch mode. The order in which the patterns are presented is randomized from one epoch to the next. This heuristic is motivated by a desire to search more of the weight space. The hyperbolic tangent function given by $(16 / 9) \tanh (2 x / 3)$, where $x$ is the input activation value, is the antisymmetric activation function used. This antisymmetric activation function is suitable for faster learning of the network [20]. Various network structures have been explored in this study. The network structure finally chosen is illustrated in Figure 2. The network is trained for 200 epochs. The block diagram of the proposed system for improving the quality of the TM speech is shown in Figure 3.

In the testing stage, the cepstral coefficients of the NM speech are estimated as described in Section 3.1. The LP spectra (LP order $=8$ ) of the test $(\mathrm{TM})$ input speech and the corresponding (desired) NM speech, and the reconstructed LP spectra are shown for various sound units in Figure 4. The reconstructed spectra are similar to the NM spectra for various sound units. It is seen that, in the case of vowels, the higher formants have a steep fall in the case of TM spectra. In contrast, the spectral roll-off in the reconstructed spectra is comparatively less, as in the NM spectra. This shows that higher formants are emphasized in the reconstructed spectra. The TM spectra for the voiced stop consonants $/ \mathrm{g} /$ and $/ d /$ resemble that of a vowel. This is due to the presence of formant-like structures during the closure phase. However, in the reconstructed spectra, as in the NM spectra, no such well-defined peaks are visible. In the case of nasals, the location of the formant(s) in the reconstructed spectra and the NM spectra differs only slightly. The oral resonance seen in the TM spectra is missing in the reconstructed spectra. It is observed that the mapping is generally not learnt well in the case of fricatives. This is because of the random noiselike signal characteristic of fricatives. The LP spectra for a sequence of frames of the TM and NM speeches, and the corresponding reconstructed spectra are shown in Figure 5. It is seen that the higher-frequency content, missing in the TM spectra is incorporated in the reconstructed spectra. It is also seen from this figure that the network is able to provide a smooth estimate of the NM spectra over consecutive frames. The advantage of this method is that no distortion (due to spectral discontinuity between adjacent frames) is perceived in the reconstructed speech.

The performance of this mapping technique is evaluated using the Itakura distance measure as the objective criterion. The Itakura distance measures the distance between two LP spectra. The Itakura distances between two LP vectors, say $\mathbf{a}_{k}$ and $\mathbf{b}_{k}$, are given by [13]

$$
\begin{aligned}
& d_{a b}\left[\mathbf{a}_{k}, \mathbf{b}_{k}\right]=\frac{\mathbf{b}_{k}^{T} \widetilde{\mathbf{R}}_{s_{a}} \mathbf{b}_{k}}{\mathbf{a}_{k}^{T} \widetilde{\mathbf{R}}_{s_{a}} \mathbf{a}_{k}}, \\
& d_{b a}\left[\mathbf{a}_{k}, \mathbf{b}_{k}\right]=\frac{\mathbf{a}_{k}^{T} \widetilde{\mathbf{R}}_{s_{b}} \mathbf{a}_{k}}{\mathbf{b}_{k}^{T} \widetilde{\mathbf{R}}_{s_{b}} \mathbf{b}_{k}},
\end{aligned}
$$

where $d_{a b}$ and $d_{b a}$ are the asymmetric distances from $\mathbf{a}_{k}$ to $\mathbf{b}_{k}$ and vice versa, respectively. $\widetilde{\mathbf{R}}_{s_{a}}=\left\{r_{s_{a}}\right\}$ and $\widetilde{\mathbf{R}}_{s_{b}}=\left\{r_{s_{b}}\right\}$, where $\left\{r_{s_{a}}\right\}$ and $\left\{r_{s_{b}}\right\}$ are the signal autocorrelation coefficients of the speech frames corresponding to $\mathbf{a}_{k}$ and $\mathbf{b}_{k}$, respectively. The symmetric Itakura distance between the two vectors is given by $d=0.5\left(d_{a b}+d_{b a}\right)$. The Itakura distance between the TM and the reconstructed spectra, and the NM and the reconstructed spectra are computed for each frame. Figure 6 shows the Itakura distance plot for an utterance. It can be observed that the distance between the NM and the reconstructed spectra is very small when compared to the distance between the NM and the TM spectra. This shows that the reconstructed spectra are very close to the NM spectra. Thus, the mapping network is able to capture the spectral correlation between the TM and NM speeches of a speaker. Listening to the reconstructed speech (speech synthesized using the estimated LP coefficients derived from the network 

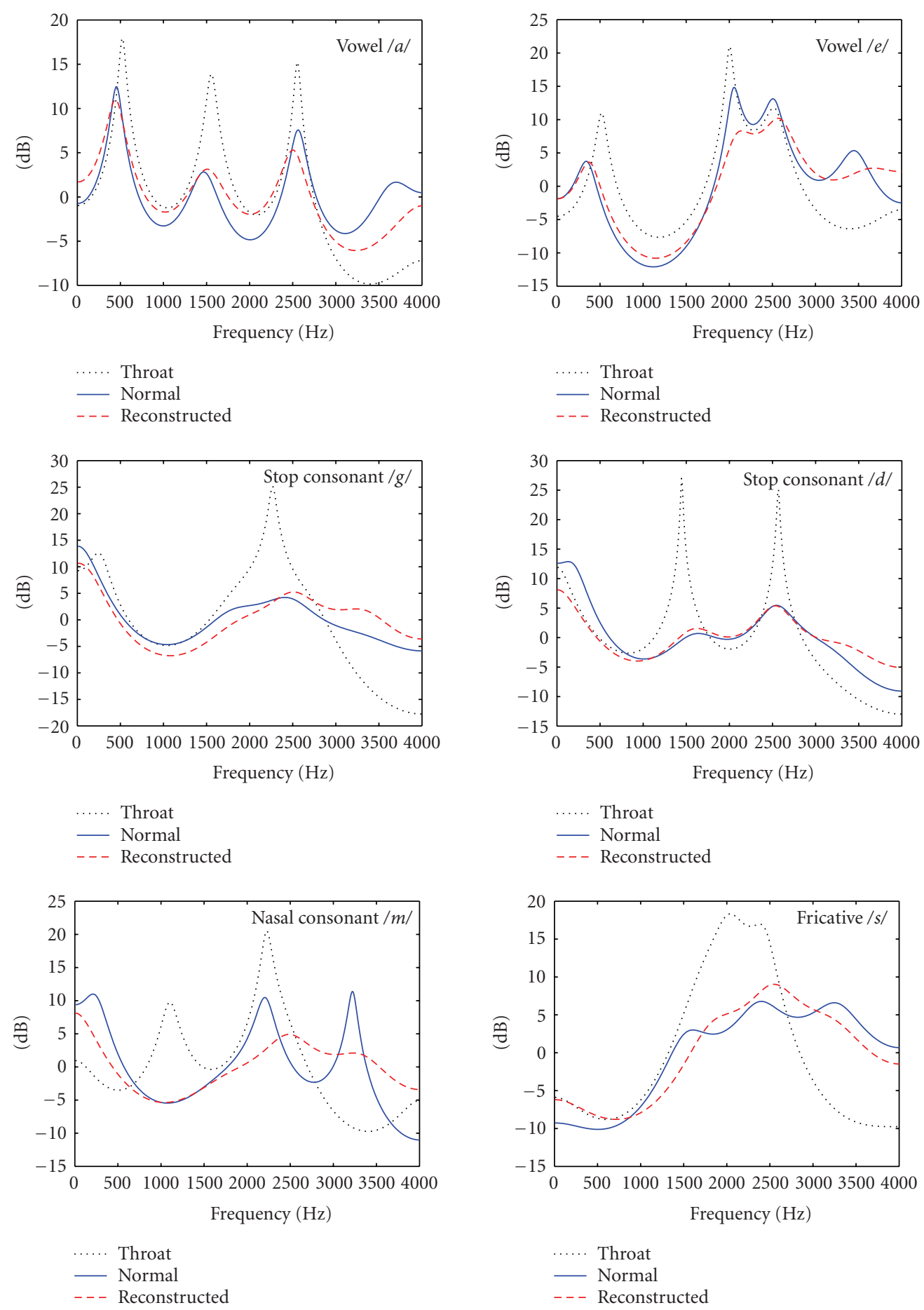

FIgURE 4: The LP spectra of the TM speech and the NM speech, and the estimated LP spectra, for the sound units $/ a /, / e /, / g /, / d /, / m /$, and $/ s$.

output and the LP residual derived from the TM speech) also shows that it sounds more natural than the TM speech.

\subsection{Bandwidth extension of telephone speech}

The mapping technique can also be used to extend the bandwidth of the narrowband $(300-3400 \mathrm{~Hz})$ telephone speech.
The data for this study comprises of speech simultaneously recorded from a normal microphone at the transmitting end, and a telephone at the receiving end. The mapping is performed using the procedure described in Section 3. Here, features from the bandlimited telephone speech form the input for the mapping network. The features of the corresponding NM speech form the target output for the network. 

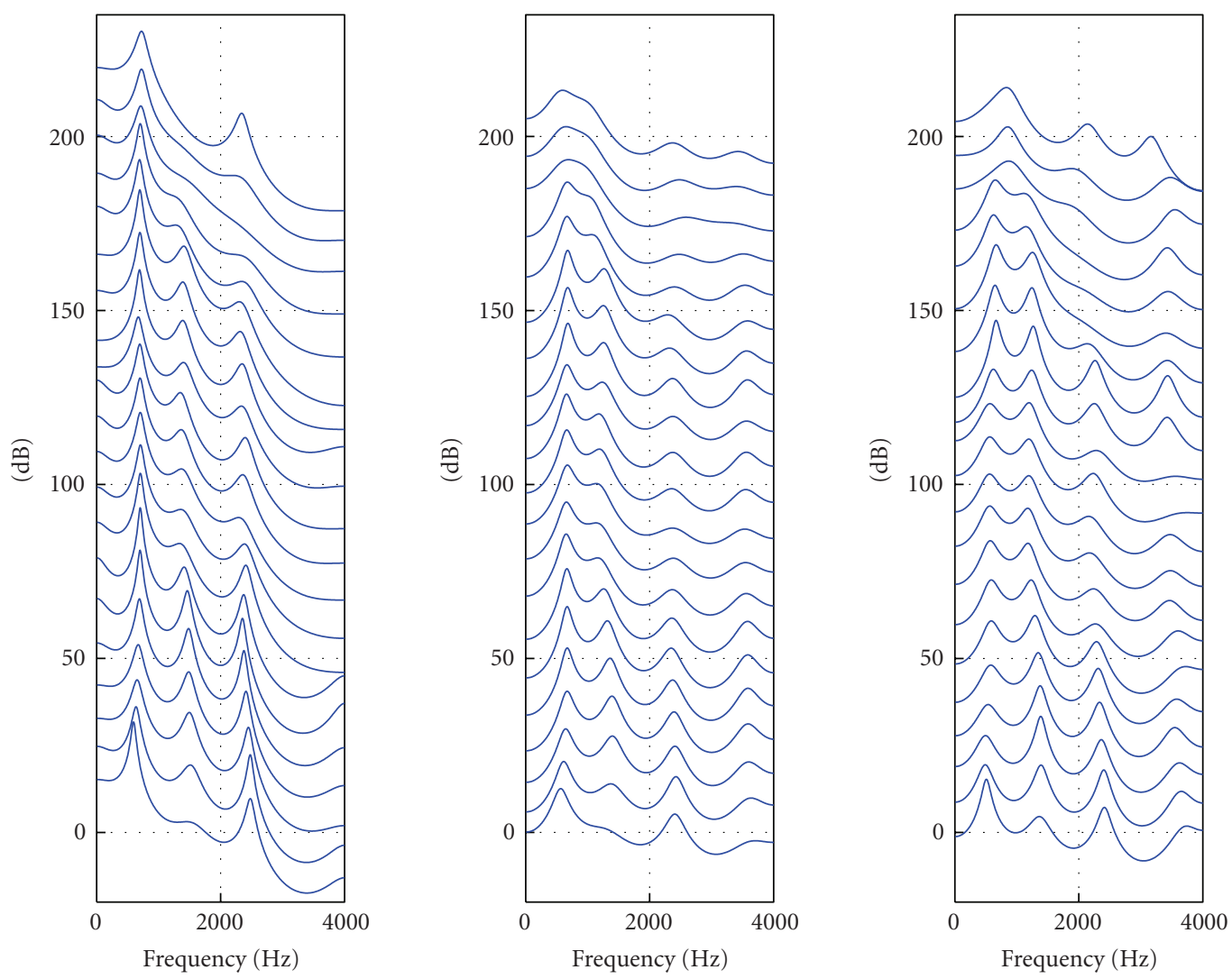

FIGURE 5: The LP spectra of the TM speech and the NM speech, and the estimated LP spectra, for a sequence of speech frames.

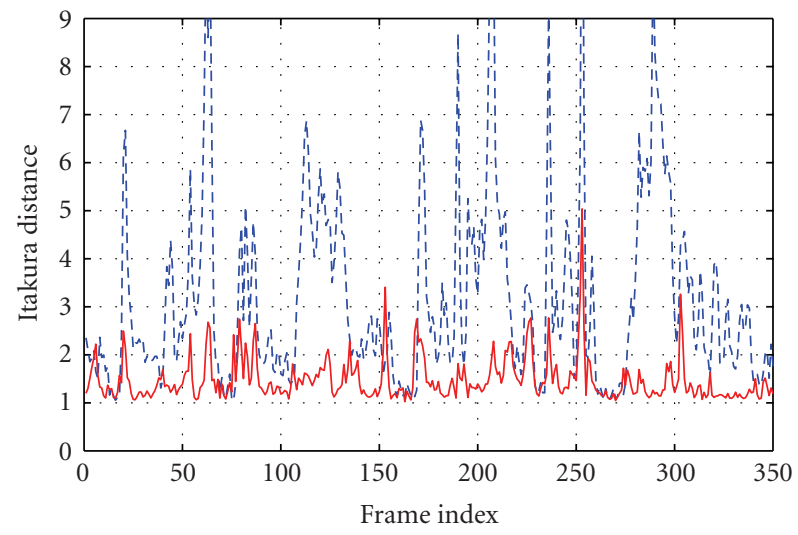

Figure 6: Itakura distance between the NM and TM spectra (dashed lines), and the NM and estimated spectra (solid lines) for a speech utterance.

In the testing stage, wideband residual regeneration is done using spectral folding approach [1]. This residual is used to excite the synthesis filter constructed from the estimated wideband LP coefficients derived from the mapping network. The LP spectra of the telephone speech, the bandwidth extended speech, and the wideband NM speech are given for two different speech frames in Figure 7. It is seen that the spectra of the bandwidth extended speech are very similar to the spectra of the wideband NM microphone speech. In this task, the issue of reconstructing the wideband LP spectra alone is addressed. It has been observed that due to the channel noise, the LP prediction error is large for telephone speech. Hence, a simple technique for regeneration of wideband residual would not suffice. Further work is necessary to manipulate the telephone residual signal for regeneration of clean, wideband residual signal. This would further improve the quality of the bandwidth extended speech.

\section{CONCLUSIONS}

A method to improve the quality of the TM speech has been proposed based on the speaker-dependent relationship between the spectral features of the TM speech and the NM speech. The mapping of the spectra has been modelled using a feedforward neural network. The underlying assumption is that the wideband NM speech is closely related to the narrowband TM speech. The stability of the all-pole synthesis filter has been ensured while estimating the features. The spectra of the reconstructed speech show that the higher frequencies that were previously of low amplitude in the TM speech are now emphasized. Thus the network was shown to capture the functional relationship between the two spectra. 

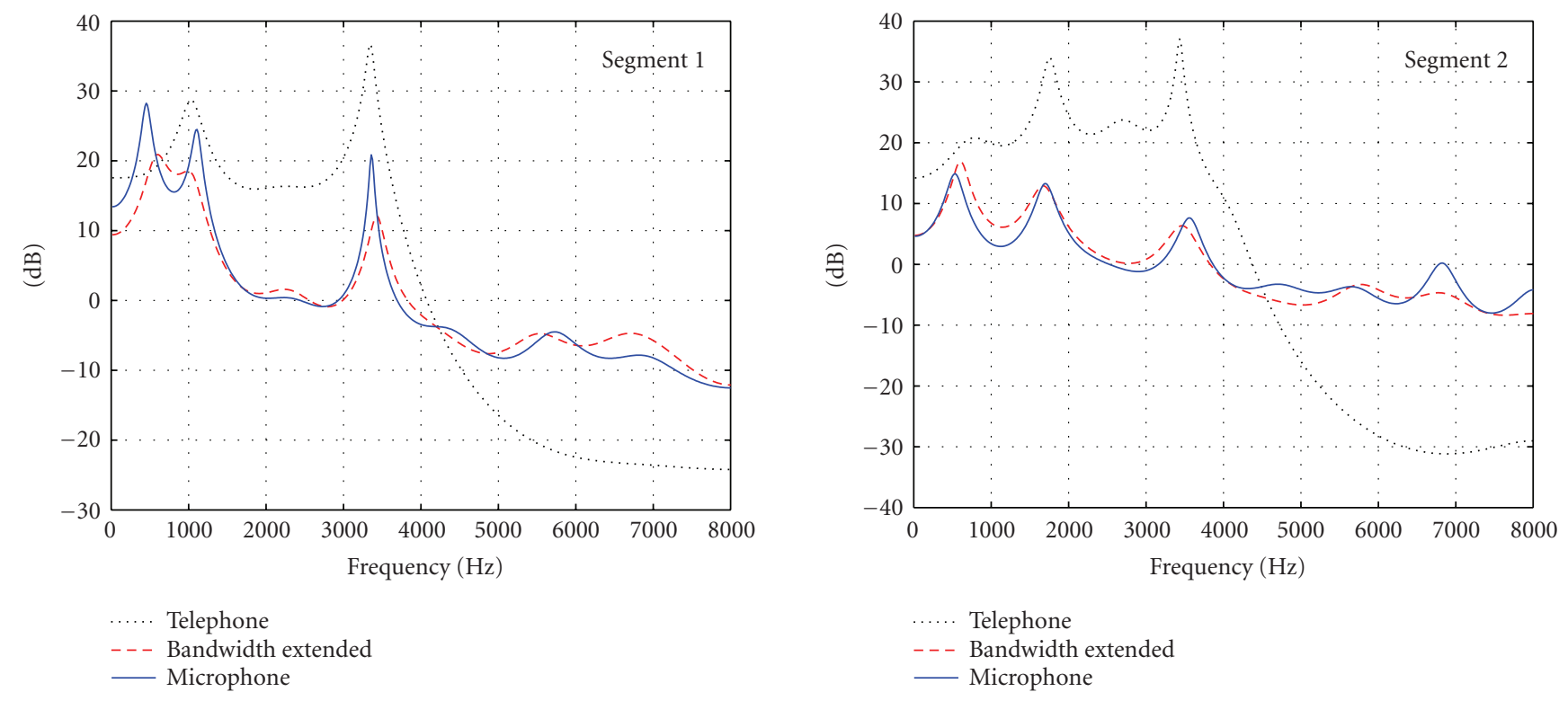

FIGURE 7: The LP spectra of the telephone speech (dotted line), bandwidth extended speech (dashed line), and NM speech (solid line) for four different segments of speech.

The advantage in this method is that distortion due to spectral discontinuities between adjacent frames is not perceived in the reconstructed speech. In this method, only the spectral features of the TM speech were modified, the excitation source features were not modified. Our future work focusses on replacing the source features of the TM speech with the source features of the NM speech in order to further improve its perceptual quality. This study shows that the proposed mapping technique can also be effectively used for the task of bandwidth extension of telephone speech. Here again, we need to address the issue of wideband regeneration of the LP residual. This would require a fresh approach, as any simple technique for high-frequency regeneration would not produce the desired result.

\section{REFERENCES}

[1] J. A. Fuemmeler, R. C. Hardie, and W. R. Gardner, "Techniques for the regeneration of wideband speech from narrowband speech," EURASIP Journal on Applied Signal Processing, vol. 2001, no. 4, pp. 266-274, 2001.

[2] R. Hu, V. Krishnan, and D. V. Anderson, "Speech bandwidth extension by improved codebook mapping towards increased phonetic classification," in Proceedings of the 9th European Conference on Speech Communication and Technology (INTERSPEECH-ICSLP '05), pp. 1501-1504, Lisbon, Portugal, September 2005.

[3] M. L. Seltzer, A. Acero, and J. Droppo, "Robust bandwidth extension of noise-corrupted narrowband speech," in Proceedings of the 9th European Conference on Speech Communication and Technology (INTERSPEECH-ICSLP '05), pp. 1509-1512, Lisbon, Portugal, September 2005.

[4] J. Makhoul and M. Berouti, "High-frequency regeneration in speech coding systems," in Proceedings of IEEE International Conference on Acoustics, Speech, and Signal Processing
(ICASSP '79), vol. 4, pp. 428-431, Washington, DC, USA, April 1979.

[5] B. Geiser, P. Jax, and P. Vary, "Artificial bandwidth extension of speech supported by watermark-transmitted side information," in Proceedings of the 9th European Conference on Speech Communication and Technology (INTERSPEECH-ICSLP '05), pp. 1497-1500, Lisbon, Portugal, September 2005.

[6] J. Epps and W. H. Holmes, "A new technique for wideband enhancement of coded narrowband speech," in Proceedings of IEEE Workshop on Speech Coding, pp. 174-176, Porvoo, Finland, June 1999.

[7] K.-Y. Park and H. S. Kim, "Narrowband to wideband conversion of speech using GMM based transformation," in Proceedings of IEEE International Conference on Acoustics, Speech, and Signal Processing (ICASSP '00), vol. 3, pp. 1843-1846, Istanbul, Turkey, June 2000.

[8] G. Chen and V. Parsa, "HMM-based frequency bandwidth extension for speech enhancement using line spectral frequencies," in Proceedings of IEEE International Conference on Acoustics, Speech, and Signal Processing (ICASSP '04), vol. 1, pp. 709712, Montreal, Quebec, Canada, May 2004.

[9] B. Iser and G. Schmidt, "Bandwidth extension of telephony speech," EURASIP Newsletter, vol. 16, no. 2, pp. 2-24, 2005.

[10] A. Uncini, F. Gobbi, and F. Piazza, "Frequency recovery of narrow-band speech using adaptive spline neural networks," in Proceedings of IEEE International Conference on Acoustics, Speech, and Signal Processing (ICASSP '99), vol. 2, pp. 9971000, Phoenix, Ariz, USA, March 1999.

[11] M. Graciarena, H. Franco, K. Sonmez, and H. Bratt, "Combining standard and throat microphones for robust speech recognition," IEEE Signal Processing Letters, vol. 10, no. 3, pp. 72-74, 2003.

[12] Z. Zhang, Z. Liu, M. Sinclair, et al., "Multi-sensory microphones for robust speech detection, enhancement and recognition," in Proceedings of IEEE International Conference on Acoustics, Speech, and Signal Processing (ICASSP '04), vol. 3, pp. 781-784, Montreal, Quebec, Canada, May 2004. 
[13] J. R. Deller, J. G. Proakis, and J. H. L. Hansen, Discrete-Time Processing of Speech Signals, Macmillan, New York, NY, USA, 1993.

[14] B. Yegnanarayana, "On timing in time-frequency analysis of speech signals," Sadhana, vol. 21, part 1, pp. 5-20, 1996.

[15] A. Shahina and B. Yegnanarayana, "Recognition of consonantvowel units in throat microphone speech," in Proceedings of International Conference on Natural Language Processing, pp. 85-92, Kanpur, India, December 2005.

[16] P. Ladefoged, A Course in Phonetics, Harcourt College Publishers, Orlando, Fla, USA, 2001.

[17] A. Shahina and B. Yegnanarayana, "Mapping neural networks for bandwidth extension of narrowband speech," in Proccedings of the 9th International Conference on Spoken Language Processing (INTERSPEECH-ICSLP '06), Pittsburgh, Pa, USA, September 2006.

[18] B. Yegnanarayana, Artificial Neural Networks, Prentice-Hall, New Delhi, India, 1999.

[19] H. Misra, S. Ikbal, and B. Yegnanarayana, "Speaker-specific mapping for text-independent speaker recognition," Speech Communication, vol. 39, no. 3-4, pp. 301-310, 2003.

[20] S. Haykin, Neural Networks: A Comprehensive Foundation, Prentice-Hall, Englewood Cliffs, NJ, USA, 1999.

A. Shahina was born in India in 1973. She graduated in 1994 from Government. College of Engineering-Salem, Madras University, India, in electronics and communication engineering. She received the M.Tech degree in biomedical engineering from Indian Institute of Technology, (IIT) Madras Chennai, India, in 1998. She was a Member of the faculty at SSN College of Engineering, Madras University, till 2001. Since 2002, she

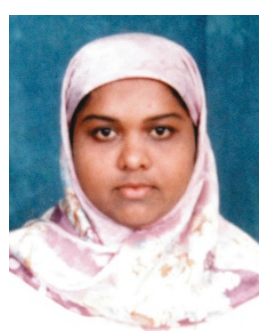
is working as a Project Officer in the Computer Science and Engineering Department at IIT-Madras, and is pursuing her Ph.D. degree. Her research interests are in speech processing and pattern recognition.

B. Yegnanarayana is a Professor and Microsoft Chair at IIIT Hyderabad. Prior to joining IIIT, he was a Professor in the Department of Computer Science and Engineering at IIT Madras, India, from 1980 to 2006. He was a Visiting Associate Professor of computer science at Carnegie-Mellon University in USA from 1977 to 1980 . He was a Member of the faculty at the Indian Institute of Science (IISc), Bangalore, from

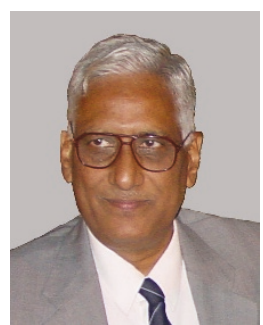
1966 to 1978. He got B.E., M.E., and Ph.D. (all in electrical communication engineering) degrees from IISc, Bangalore, in 1964, 1966, and 1974, respectively. His research interests are in signal processing, speech, image processing, and neural networks. He has published over 300 papers in these areas in IEEE and other international journals, and in the proceedings of national and international conferences. He is also the author of the book "Artificial Neural Networks," published by Prentice-Hall of India in 1999. He has supervised 21 Ph.D. and 31 M.S. theses. He is a Fellow of the Indian National Academy of Engineering, a Fellow of the Indian National Science Academy, and a Fellow of the Indian Academy of Sciences. He was the recipient of the 3rd IETE Professor S. V. C. Aiya Memorial Award in 1996. He received the Professor S. N. Mitra Memorial
Award for the year 2006 from the Indian National Academy of Engineering for his significant and unique contributions in speech processing applications, and for pioneering work in teaching and research in signal processing and neural networks. 